2

\title{
Intercontinental genomic parallelism in multiple adaptive radiations
}

Isabel S. Magalhaes*1,2, James R. Whiting*1,3 , Daniele D'Agostino ${ }^{1}$, Paul A. Hohenlohe ${ }^{4}$, Muayad Mahmud ${ }^{1}$, Michael A. Bell ${ }^{5}$, Skúli Skúlason ${ }^{6}$ and Andrew D.C. MacColl ${ }^{1}$

${ }^{1}$ School of Life Sciences, University of Nottingham, University Park, Nottingham, NG7 2RD, U.K.

${ }^{2}$ Department of Life Sciences, Whitelands College, University of Roehampton, London SW15 $4 J D$, U.K.

${ }^{3}$ Biosciences, College of Life and Environmental Sciences, Geoffrey Pope, University of Exeter, Stocker Road, EX4 4QD, U.K.

${ }^{4}$ Institute for Bioinformatics and Evolutionary Studies, Department of Biological Sciences, University of Idaho, Moscow, ID 83844, USA.

${ }^{5}$ Department of Ecology \& Evolution, Stony Brook University, 650 Life Sciences Building, Stony Brook, NY 11794-5245

${ }^{6}$ Department of Aquaculture and Fish Biology, Hólar University College, Saudárkrókur, Iceland

*These authors contributed equally to this work

\section{Abstract}

Parallelism, the evolution of similar traits in populations diversifying in similar conditions, provides good evidence of adaptation by natural selection. Many studies of parallelism have focused on comparisons of strongly different ecotypes or sharply contrasting environments, defined a priori, which could upwardly bias the apparent prevalence of parallelism. Here, we estimated genomic parallelism associated with individual components of environmental and phenotypic variation at an intercontinental scale across four adaptive radiations of the three-spined stickleback (Gasterosteus aculeatus), by associating genome-wide allele frequencies with continuous distributions of environmental and phenotypic variation. We found that genomic parallelism was well predicted by parallelism of phenotypeenvironment associations, suggesting that a quantitative characterization of phenotypes and environments can provide a good prediction of expected genomic parallelism. Further, we examined the explanatory power of genetic, phenotypic, and environmental similarity in predicting parallelism. We found that parallelism tended to be greater for geographically 
34 proximate, genetically similar radiations, highlighting the significant contingency of standing

35 variation in the early stages of adaptive radiations, before new mutations accumulate.

36 However, we also demonstrate that distance within multivariate environmental space

37 predicts parallelism, after correction for genetic distance. This study thus demonstrates the

38 relative influences of environment, phenotype and genetic contingency on repeatable signatures of adaptation in the genome.

\section{Introduction}

42 Adaptive radiations are well known as rapid branchings on the tree of life $\left({ }^{1}\right)$. They may be

43 the source of most biodiversity, and their study has revealed a great deal about the

44 evolution of phenotypic diversity $\left({ }^{1,2}\right)$. However, patterns in adaptive radiations also

45 highlight some of the unknowns about how biodiversity evolves. For example, although

46 adaptive radiations are typified by abundant phenotypic diversity, not all trait combinations

47 evolve in every radiation, while on the other hand organisms in different places sometimes

48 arrive at very similar endpoints $\left({ }^{3,4}\right)$. This suggests that Stephen Jay Gould's famous

49 contention that evolution is contingent and unrepeatable $\left({ }^{5}\right)$ cannot be completely true. A

50 good deal of work has focussed on the role that genetic correlations between traits might

51 play in creating constraints on diversity, but so far the answers provided by this approach

52 have not been entirely satisfactory $\left({ }^{3,6,7}\right)$. Alongside these processes, it is probable evolution

53 can be shaped in a predictable way by common environments and shared selective regimes,

54 and that the emergence of repeatable patterns of evolution, a process predominantly

55 known as parallelism (which we distinguish from convergence here by the inclusion of

56 shared evolutionary 'start', as well as 'end', points, although see $\left({ }^{8,9}\right)$, is the direct result of

57 environmental similarities within and between radiations. Striking examples of phenotypic 
58 parallelism $\left({ }^{1,10}\right)$ in the natural world support this hypothesis, and the persistent appearance

59 of familiar forms in similar ecological niches demonstrates the significance of selection in

60 this process.

61 However, a significant problem with studies that have focused on phenotypic

62 parallelism is that they have concentrated largely on the comparison of pairs of strongly

63 different ecotypes or widely different environments $\left({ }^{11-13}\right)$. Such studies could upwardly bias

64 the apparent prevalence of parallelism because the chosen comparisons were known $a$

65 priori to occur repeatedly in different locations, effectively constraining the evolutionary

end point. The approach also conceals the role of individual components of environmental

variation in driving parallelism, as similar environments are often assumed based on

comparable phenotypes. This gap surely needs addressing for a complete understanding of adaptation $\left({ }^{14}\right)$. In addition, traits that are measurable in sufficient numbers from (usually) wild organisms are generally limited to morphological and life history traits $\left({ }^{13}\right)$. This measures of phenotype and environment alongside genomics in studies of parallelism. The detection of consistent genomic signatures across multiple, independent natural populations has proven to be a valuable tool for studies of evolutionary patterns and the discovery of genes involved in adaptation $\left({ }^{15-17}\right)$. However, yet again our comprehension of the relationship between genomic parallelism and continuous phenotypic or environmental variation is surprisingly poor. 
sequencing costs, such genomic data can nowadays be used alongside ecological data to associate allele frequencies $\left({ }^{18}\right)$, and determine genomic regions associated with individual components of environment and phenotypes. Such an approach is essential if studies of parallelism are to shift from description to hypothesis testing, but has rarely been applied (but see ${ }^{12,19,20}$ ), and it remains to be shown whether signals of parallelism obtained from continuous measures are comparable to those from ecotypes and from previous studies. Here we make use of such methods to test for environmentally and phenotypically associated genomic parallelism across radiations of three-spined stickleback fish (Gasterosteus aculeatus, hereafter 'stickleback').

Stickleback provide a powerful natural experiment to test parallelism. They are primitively marine but have undergone replicated adaptive radiations across the northern hemisphere following colonisation of freshwater in widely separated geographical locations. This allows us to compare multiple populations derived from the marine ancestor, and provides a model for exploring both phenotypic parallelism and its genetic basis $\left({ }^{21,22}\right)$ in response to environmental variation. Phenotypic parallelism in populations that have evolved independently in similar habitats is well established $\left({ }^{23,24}\right)$, and whilst often considered in dichotomous pairings of marine - freshwater, benthic - limnetic, lake -stream ecotypes, there is a huge amount of continuous phenotypic variation among freshwater populations that has hitherto rarely been explored $\left({ }^{25}\right)$ in this context. In addition, genome scans have identified loci that have come repeatedly under selection across the contrasting ecotype pairs $\left({ }^{17,26,27}\right)$, but the combination of the phenotypic and genomic parallelism in one study is rare (although see ${ }^{28}$ ) and has not previously been done at the scale of replicated adaptive radiations across continents. 
(Fig. 1, Supplementary Table 1), measured a set of six biotic and abiotic environmental

rakers) (Supplementary Table 2), and performed a genome-wide scan of a total of 1,304

individual stickleback using restriction-site associated DNA (RAD) sequencing. We first

at the presence of allele frequency associations in the same genomic regions across

Finally, we examined how the prevalence of parallel genomic regions is associated with the adaptation. 
Interpreting inter-radiation patterns of genomic parallelism associated with phenotypic traits and environmental variables requires that we first assess the environmental and phenotypic similarity across all four radiations analysed here, as well as the relationship between the two types of variables. Environmental similarity is predicted to influence both phenotypic and genomic parallelism, as similar selection regimes are imposed on organisms $\left({ }^{13,30,31}\right)$. These analyses provide insights into some major factors that are likely to influence the emergence of a common pattern of genomic divergence at such a large geographical scale, and indicate how much genomic parallelism associated with environments and phenotypes to expect.

I) Environment. Adaptation of stickleback to freshwater in these radiations has happened rapidly, over the course of the past few thousand years, and has most likely been driven by strong selection favouring pre-existing alleles present at low frequencies in the ancestral marine population $\left({ }^{32}\right)$. Therefore, we might expect to observe shared features of diversification when multiple groups of organisms experience similar environmental circumstances, as similar selection regimes are imposed on organisms $\left({ }^{12,33}\right)$. A Principal Component Analysis (PCA) on six measures of water chemistry ( $\mathrm{pH}$, calcium (Ca), sodium (Na) and zinc (Zn), and parasitism (prevalence of the parasites Gyrodactylus spp. (Gyro) and Schistochephalus solidus (Schisto)) across all lakes revealed that the first axis of environmental variance (Env $\left.\mathrm{PC}_{\mathrm{P} 1}\right)$ separated lakes along a gradient of $\mathrm{pH}$ and calcium concentration (Fig. 2; Supplementary Table 3). This axis did not separate radiations but emphasised the variation from alkaline to acid present in all of them, with the widest range in Alaska. The second axis of environmental variance $\left(E_{n v_{P C 2}}\right)$ distinguished mostly between lakes with high and low zinc and largely separated the European from the North American 
152 lakes. The two groups of European lakes overlapped very little environmentally with each

153 other along the second axis or with the North American lakes, but the variation in BC lakes

154 was completely subsumed within that of the more environmentally variable Alaskan lakes.

155 An analysis of the similarity of the direction of the major PC vectors $(\theta)$ of

156 environmental variation (PCs that explained at least $10 \%$ of the variation) and of the

157 magnitude of their variation revealed that Alaska and $\mathrm{BC}$ exhibited the strongest

158 environmental similarity in terms of direction $\left(\theta=8.71^{\circ}, p=0.077\right)$ (Supplementary Table 4),

159 as lower values of $\theta$ are indicative of more common environmental covariance. However,

160 they also showed the greatest difference in magnitudes of variation, as BC had the lowest

161 amount of environmental variation of all radiations while Alaska had the highest. Iceland on

162 the other hand was the most unique radiation in terms of environmental covariance, a

163 pattern likely driven by Iceland's uniquely volcanic nature.

II) Phenotypes - Armour. The major axis of armour variation (Armour $\mathrm{PC}_{\mathrm{PC}}$ ) represented

a correlated axis of all traits, particularly dorsal spine length and pelvic characteristics (Fig.

166 2; Supplementary Table 3). Although there were significant differences in Armour ${ }_{\mathrm{PC} 1}$ among

167 both radiations and lakes (Supplementary Table 5), the direction of this vector was highly

168 conserved across all radiations $\left(4.13^{\circ} \leq \theta \leq 8.37^{\circ} ; 0.004 \leq p \leq 0.053\right)$ (Supplementary Table

169 4). Nonetheless, we found populations with extreme armour, such as absence or extreme

170 reduction of dorsal spines, pelvic spines, pelvis size, and generally low Armour $_{\mathrm{PC} 1}$, that occur

171 in Scotland and Alaska but not elsewhere. However, while in Scotland pelvic armour

172 reductions were accompanied by complete or almost complete loss of armour plates (high

173 Armour $\left.\mathrm{PC}_{2}\right)$, Alaskan populations retained those even when other features of armour were

174 highly reduced. These deviations produce the somewhat anomalous relationship between

175 Armour $_{\mathrm{PC} 1}$ and Armour $\mathrm{PC}_{\mathrm{PC}}$ in Alaska (Fig. 2). Iceland exhibited particularly low variation in 
176 armour traits compared to other radiations, while $\mathrm{BC}$ had the highest. These results indicate

177 that whilst the direction of armour variation occurred predominantly on a shared axis across

178 adaptive radiations, the amount of armour variation along that axis was variable. When

179 comparing all freshwater populations with four marine ones (one from each country), we

180 found that, aside from a few populations from BC, there was no overlap in armour traits

181 between marine and freshwater populations. This is because marine populations have a

182 higher number of lateral plates and more exaggerated armour traits in general. Importantly

183 however, projection of marine armour phenotypes suggests they fall on the same axis but

184 beyond freshwater space (Fig. 2).

III) Phenotypes -Body shape. Despite some overlap in body shape across all radiations

suggesting similar morphologies have evolved repeatedly across continents (Fig. 2), there

were significant differences in body shape morphologies among both radiations and lakes

(Supplementary Table 5). The most extreme body shapes were found in Scotland, where

some populations have very elongated and slender bodies with small heads (Shape $\mathrm{PC1}_{\mathrm{P}}$ axis),

and in $\mathrm{BC}$ where some populations had the deepest bodies and heads and the longest heads

(Shape $_{\mathrm{PC2}}$ axis). Scotland had the largest amount of variation in body shape, which is a

surprising result given this variation is found within $1000 \mathrm{~km}^{2}$ of North Uist, a much smaller

area than the other three radiations. The orientation of the major PC vectors $(\theta)$ revealed

that North American radiations were the most $\operatorname{similar}\left(\theta=3.49^{\circ}, p=0.19\right)$, followed by the

European radiations $\left(\theta=8.48^{\circ}, \mathrm{p}=0.63\right.$ ) (Supplementary Table 4), which highlights a 
200 length was found in Alaska, while BC populations contained a subset of that variation (Fig.

201 2). Across the two dimensions of gill raker number and length, variation within radiations

was generally constrained to a major axis in which populations with more gill rakers also

tended to have longer gill rakers. All freshwater populations generally had shorter gill rakers

for their size relative to marine populations.

V) Relationship between environmental and phenotypic similarity. We used GLMs to

test for parallel associations between the first two environmental PCS (Env $v_{P C 1}$ and Env $v_{P C 2}$ )

and armour, shape, and gill raker morphology across radiations. We found that Armour PC1

and Armour $_{\mathrm{PC2}}$ were also significantly associated with Env $_{\mathrm{PC} 1}$ (Armour $_{\mathrm{PC1}}$ : $F=6.13, p=0.016$;

ArmourPC2: $F=4.11, p=0.047$ ), but not with $E v_{P C 2}$ (Armour $_{P C 1}: F=1.12, p=0.293$; Armour $_{P C 2}$ :

$\mathrm{F}=1.10, \mathrm{p}=0.298$ ) (Supplementary Table 6). Armour ${ }_{\mathrm{PC} 1}$ correlations with Env PC1 $_{\mathrm{P} 1}$ were similar

across radiations but significant slope variation was observed between radiations for

$\operatorname{Armour}_{\mathrm{PC} 2}$ and $\mathrm{Env}_{\mathrm{PC} 1}$ associations $(\mathrm{F}=2.76, \mathrm{p}=0.050)$. The result highlights parallelism in the

way general skeletal traits (Armour ${ }_{\mathrm{PC} 1}$ ) are reduced with lower $\mathrm{pH}$ and calcium (Env $\mathrm{PC}_{\mathrm{P} 1}$ ), but

non-parallel associations between these measures and the relationship between pelvic size

and plate number (Armour $\mathrm{PC}_{2}$ ). The latter is consistent with previous findings of a lack of

radiations $\left({ }^{34}\right)$, while the former suggests that common $\mathrm{pH}$ and calcium environments,

experienced in all radiations as a shared acid-alkali axis, promotes phenotypic parallelism. 
223 indicating parallelism across radiations in the way shape and environment are associated

224 (Supplementary Table 6). The result highlights parallelism in the way bodies become more

225 elongated and slender and heads are reduced with lower $\mathrm{pH}$ and calcium and higher Zinc.

226 As for gill rakers, we expected trophic morphology to evolve in response to 227 zooplankton communities, which in turn have been shown to vary in response to measures

228 of water chemistry $\left({ }^{35,36}\right)$, in particular $\mathrm{Zn}$ which was the dominant component of Env $\mathrm{PC2}$.

229 Consistent with this, we found gill raker number to be strongly significantly associated with 230 both $\operatorname{Env}_{P C 2}(F=43.74, p<0.001)$ and $\operatorname{Env}_{P C 1}(F=18.17, p<0.001)$. Slopes varied between 231 radiations for the association of gill raker number and Env $v_{\mathrm{PC} 1}$, demonstrating non-parallelism 232 in trophic response to $\mathrm{pH}$ and calcium. In contrast, common slopes across radiations 233 highlight parallelism in trophic association between gill raker number and $\mathrm{Zn}$ variation $234\left(\mathrm{Env}_{\mathrm{PC2}}\right)$.

235 Corroborating predictions that selection imposed by similar environments can lead 236 to phenotypic parallelism $\left({ }^{1}\right)$, we found that similar variation from alkaline to acid 237 environments (Env $\mathrm{PC}_{\mathrm{P}}$ ) present in all radiations was significantly associated with parallel 238 variation in body shape and armour. If phenotypic adaptation happens via natural selection 239 on genetic variation, we would then expect these associations to translate into genomic 240 parallelism associated with both environmental variables that show a similar range across radiations ( $\mathrm{pH}$ and $\mathrm{Ca}$ ) and phenotypic traits associated with them.

\section{Phylogenetic relationship among radiations.}

244 The probability of genomic parallelism has been linked to time since lineages split,

245 highlighting the probable influence of genetic similarity and shared ancestral variants on 
246 parallelism $\left({ }^{16}\right)$, it is therefore important that we know the genetic relationship across all

247 populations before interpreting patterns of genomic parallelism. Genomic parallelism across

248 populations within any one radiation is likely to evolve from shared genetic variation, but

249 the probability of that should decline in geographically distant radiations as a function of

250 common ancestry $\left({ }^{16,37}\right)$. Indeed, recent studies have highlighted the probable limitation of

251 parallelism in this system across continental scales $\left({ }^{20,38,39}\right)$. A Neighbour Joining (NJ) tree

252 based on 8,395 unlinked SNPs showed that, with the exception of a likely recently formed

253 population in Alaska (TERN), which is basal to both the Alaskan and British Columbian

254 radiations, the four geographic locations form four well-resolved radiations (Fig. 1). A PCA

255 on the same dataset confirmed that radiations form independent clusters, and also revealed

256 that the dominant axis of variation (PC1 $=36.0 \%)$ separates North American and European

257 radiations pairs from one another (Supplementary Fig. 1). North American radiations then

258 separated on PC2 (7.0\%) and European radiations on PC3 (5.8\%). In addition to being close

259 together in the NJ tree, we found that the geographically adjacent radiations were also the

260 most genetically similar (Supplementary Table 7): Alaska and BC (mean pairwise $F_{S T}=0.198$ ),

261 and Scotland and Iceland $\left(F_{S T}=0.194\right)$, suggesting that although these form independent

262 clusters, the lineage split between them is relatively recent, or that any gene flow between

263 radiations is occurring within the Atlantic and Pacific groups. Genetic divergence between

264 inter-continental pairings was found to be stronger and deeper (0.314 ? $\mathrm{F}_{S T}$ ? 0.338) than

265 within continents, which is consistent with previous studies that have estimated the time of

266 divergence between stickleback from Europe and North America to be approximately

267200,000 years $\left({ }^{40,41}\right.$ but see $\left.{ }^{42}\right)$. Further, between-continent structuring accounts for the

268 largest proportion of molecular variance in our data (AMOVA: $\sigma=889.7,34.7 \%$ ). Within

269 continents, populations within radiations ( $\sigma=366.5,14.3 \%)$ were more genetically variable 
270 than radiations ( $\sigma=142.3,5.6 \%$ ) (Supplementary Table 8 ). This highlights that molecular

variance isn't structuring according to geographic scale (Continent > Radiation >

272 Populations), but rather gene flow may be occurring between intra-continental radiations.

273 Following the idea that the probability of parallelism at the genetic level is linked to

274 time since lineages split (i.e. genetic similarity), we would expect groups with closer

275 evolutionary histories, or indeed present gene flow, to show the strongest genomic

276 parallelism if adaptation occurs through shared standing genetic variation or the exchange

277 of beneficial freshwater alleles between intra-continental radiations via marine populations

$278 \quad\left({ }^{29,32}\right)$

279

Phenotypically and environmentally associated SNPs and genomic regions within

radiations.

282 We scanned the genome to identify regions associated with individual components of

phenotypic and environmental variation within each of the four adaptive radiations. We

focused on repeated changes within the same genomic regions, rather than on reuse of the

same mutations. This is because the causal mutations are unknown in most cases and may

not be sequenced by reduced representation sequencing methods such as RAD-sequencing.

For each radiation separately ( $\mathrm{N}=18$ or 19 populations) we used Bayesian linear models

288 implemented in Bayenv2 $\left({ }^{43}\right)$ to identify associations between population SNP allele

289 frequencies ( $\mathrm{N}=10$ to 21 individual fish, mean= 17.8), the set of six biotic and abiotic

290 environmental variables and the set of 12 phenotypic traits (Supplementary Table 2). As a

291 positive control for the methods used we compared our results for parallelism across

292 freshwater radiations with well-studied marine-freshwater parallelism in this species

$293\left({ }^{17,27,29}\right)$, and then examined genomic differentiation between all freshwater populations 
294 pooled within a radiation and four marine populations pooled together (one from each

295 country, Supplementary Table 1).

296 Our analyses identified population allele frequencies of several thousand SNPs for

297 each radiation as being highly correlated (high Bayesfactor $\left[>\log _{10}(1.5)\right]$ and top $5 \%$ of

298 Spearman's $\rho$, see methods) with the abiotic and biotic environmental variables

299 ('environmentally associated SNPs') or with phenotypic traits ('phenotypically associated

SNPs' (Supplementary Table 9). SNPs were then mapped onto non-overlapping sliding

windows of $50 \mathrm{~kb}, 75 \mathrm{~kb}, 100 \mathrm{~kb}$ or $200 \mathrm{~kb}$, which allowed us to test the robustness of our

results across different extents of linkage. Further, we repeated our analysis across windows

of equivalent genetic distance $(0.1 \mathrm{cM}$ windows), which confirmed that our results were not

influenced by variable linkage across the genome (Supporting Information). Here we report

only results for $50 \mathrm{~kb}$ windows, given that this is consistent with approximate linkage

disequilibrium within the stickleback genome $\left({ }^{44,45}\right)$. Results for window sizes of $75 \mathrm{~kb}$,

$100 \mathrm{~kb}, 200 \mathrm{~kb}$ can be found in Supplementary Dataset 1 . Our $50 \mathrm{~kb}$ dataset was composed of

4,868 windows with SNPs in all locations, covering approximately $55 \%$ of the $447 \mathrm{Mb}$

genome, with a further 1,940 windows sequenced in 2 or more locations providing

information on an additional $21.7 \%$ of the genome.

Windows were classified as 'environmentally associated' and 'phenotypically 
environmental variables, even when their variation is modest, clearly supporting the adaptive nature of these radiations.

phenotypic variable in the same radiation, suggesting several regions associated with

322 (Supplementary Dataset 1). It also suggests that directly measuring important aspects of the phenotypes.

\section{Genomic parallelism associated with environmental and phenotypic variation across}

radiations.

To quantify genomic parallelism we identified environmentally- and phenotypicallyassociated windows that were shared across two or more of our radiations, i.e. parallel windows (Supplementary Fig. 2). We then compared the overall observed numbers of parallel windows to a null distribution of randomly associated windows permuted over 10,000 iterations. parallelism associated across all radiations for groups of phenotypes or environment; 2) genomic parallelism associated with individual variables across all comparisons to understand contributions of individual variables; 3) parallelism for individual variables in specific pairings, to identify pairs of radiations having the highest levels of parallelism, and for which variables. The latter is important as patterns of parallelism may be lost when we 
pool more than two radiations or variables together, if for example parallelism is very strong in one radiation-pairing but not others.

When quantifying the overall level of genomic parallelism associated with groups of environmental or phenotypic variables we found no environmentally- or phenotypicallyassociated 50kb windows parallel in all four radiations for individual variables (Randomised permutations $\mathrm{N}_{\text {Expected-Environmental }}=0.0002, \mathrm{~N}_{\text {Exp-Pheno }}=0.0001$ ), but one window was parallel in a group of three radiations (chrlV: $14400000-14450000$ associated with length of pelvis in $B C$, Iceland and Scotland) $\left(\mathrm{N}_{\text {Exp-Env }}=0.05, \mathrm{~N}_{\text {Exp-Pheno }}=0.161, p=0.149\right)$. Many windows however exhibited parallelism between pairs of two radiations. A total of 39 environmentally associated windows (pooled across all 6 environmental variables) $\left(\mathrm{N}_{\mathrm{Exp}}=\right.$ 11.9, 95\% Upper limit $(U L)=18, p<0.001)$ and 65 phenotypically associated windows (pooled across all 12 phenotypic variables) $\left(\mathrm{N}_{\mathrm{Exp}}=30.9,95 \% \mathrm{UL}=40, p<0.001\right)$ were parallel between two radiations (Supplementary Fig. 2). Parallelism was disproportionately greater for armour and gill raker traits (number mostly) than for shape, with the 65 phenotypically associated windows split into 46, 12 and seven associated windows for armour, gill raker and shape variables respectively $\left(\chi^{2}=6.506, P=0.04\right)$. This is consistent with the fact that skeletal traits, several of which are known to have simple genetic architectures $\left({ }^{26,47,48}\right)$, are particularly likely to show evidence of phenotypic parallelism. Interestingly, parallel associated windows (mean SNP N $=7.13$ ) had on average more SNPS per window than non-parallel windows (mean SNP N $=6.27)\left(\mathrm{GLM}, \mathrm{LRT}_{1,3867}=22.1, p<\right.$ 0.001 ) and exhibited slightly stronger signals of association with variables (mean residual SNPs above expected $=1.82$ parallel; 1.61 non-parallel; $\mathrm{GLM}, \mathrm{LRT}_{1,3867}=5.05, p=0.025$ ). Random permutations indicated that there were statistically significant levels of parallelism for the number of windows ('significantly parallel windows') associated with two 
environmental variables ( $\mathrm{Ca}$ and $\mathrm{pH}$ ) (Fig. 3; Supplementary Table 10). Consistent with our

expectations from patterns of environmental parallelism, these were the same variables

that share an axis of variation (Env $\left.v_{\mathrm{PC} 1}\right)$ across freshwater environments in all radiations. In

addition, we did not detect significant genomic parallelism associated with variables that

exhibit variation between radiations, such as salinity, zinc and S. solidus prevalence. These

results together confirm that common environmental axes, such as the one experienced in

all radiations as a shared acid-alkali axis, likely promote signals of parallelism in the genome.

We also found more genomic parallelism than expected by chance associated with a

total of five phenotypic variables (Fig. 3; Supplementary Table 10): four armour traits ( $2^{\text {nd }}$

dorsal spine, pelvic spine length, length of pelvis and armour plate number) and gill raker

number. These results are consistent with the high heritability of skeletal traits $\left({ }^{26,47,48}\right)$, and

suggest that variation in armour is the result of different genotypes being selected in

different environments. Further, parallel QTLs have been described for gill raker number

$\left({ }^{49}\right)$, but not length, which exhibits more plasticity $\left({ }^{50}\right)$. Body shape traits were not associated

with any significant genomic parallelism, despite parallelism across radiations in the way

shape is associated with the environment. It is probable the partly plastic nature of body

shape $\left({ }^{51,52}\right)$ leads to an association between environment and body shape via the reaction

norm rather than genomic re-use.

Our analyses also showed much stronger parallelism across marine-freshwater (MxF)

comparisons than across freshwater variables (Fig. 3), and 44/158 of MxF associated

windows overlapped with previously identified genomic regions contributing to marine-

freshwater divergence $\left({ }^{17,44}\right)$ (Supplementary Table 11). Several regions found parallel for

MxF analyses were also parallel for $\mathrm{Ca}, \mathrm{pH}, \mathrm{Na}$, armour traits and gill raker number 
robust enough to recover known parallel regions, and interestingly that genomic parallelism associated with freshwater variables is more modest than marine-freshwater parallelism.

390 The latter likely reflects subtler variation between habitats within radiations in comparison to stark marine-freshwater contrasts. Further, these results highlight that binary ecotype pairings, which likely include variation in many environmental and phenotypic traits, lump together parallelism of many components of fitness without being able to discern which are parallel and which are non-parallel. Overall, our results suggest that across these four freshwater adaptive radiations, evolution of these phenotypes and environmentally associated traits are disproportionately linked to the same genomic regions.

Within specific pairings, we found the greatest number of significantly parallel windows in the comparison between Alaska and BC (two environmental variables: Ca and Gyrodactylus spp., and three phenotypic traits: pelvic spine length, plate number and gill raker number), followed by Iceland and Scotland (two environmental variables: $\mathrm{Ca}$ and $\mathrm{pH}$, and one phenotypic trait: dorsal spine length) (Supplementary Fig. 2; Supplementary Table 10). Two environmental variables and one phenotypic trait were also associated with significantly more parallel windows in comparisons between Iceland and the North American radiations: Ca and length of the pelvis (Alaska and Iceland) and Schistocephalus solidus ( $\mathrm{BC}$ and Iceland). Pelvic spine length was the only variable of any category found to be parallel between Scotland and BC, and no significant parallelism was detected between 407 Scotland and Alaska (suggesting observed overlap may be the result of chance). Phylogenetic patterns and the segregation of molecular variance strongly support the notion that radiations within continents share similar genetic variation, making parallelism through shared standing variation the most parsimonious explanation for our intra- 
411 continental parallelism biases. Experimental studies with stickleback have demonstrated

412 rapid morphological adaptation from standing genetic variation, even recovering diverse

413 morphologies from variation found within phenotypically-derived freshwater populations

$414\left({ }^{53}\right)$. Coancestry patterns, centred at the focal, causative loci, can discern between whether

415 parallel evolution occurs on de novo mutations, standing variation, or introgressed alleles,

416 however we lack the sequencing resolution in our current data to make these comparisons.

418 Linkage and the genomic location of parallel regions.

As with any reduced-representation genomic approach, the power of RAD

sequencing to detect loci associated with phenotypic or environmental variables depends

varies widely across organisms and within genomes, but has been relatively well-

423 characterized in stickleback $\left({ }^{56,57}\right)$, and RAD sequencing has been used successfully in this

424 species specifically to test for genomic parallelism $\left({ }^{30,44,53,56,57}\right)$. One explanation for variable

425 linkage across the genome is the negative relationship it shares with local recombination

426 rate. To examine its influence on our results, we estimated recombination rate using a

427 previously published genetic map $\left({ }^{31}\right)$ for our $50 \mathrm{~kb}$ windows, and marked windows that were

428 associated with any variable and associated windows that were also parallel across

429 radiations. Recombination was significantly reduced in associated windows and parallel

430 windows compared with non-associated windows (Supplementary Fig. 3; Kruskal-Wallis, $\chi^{2}=$

$431121.43, p<0.001)$, but did not differ significantly between outlier and parallel windows $(p=$

432 0.55). Reduced recombination can be an important process in adaptation through 
433 maintaining adaptive alleles, as has been demonstrated in this species $\left({ }^{58}\right)$ and others (eg.

$\left.434{ }^{59}\right)$. However, we cannot rule out that these patterns are produced by an increased ability to

435 detect selection in low-recombination windows as a product of increased linkage with

436 causative SNPs. The latter suggests that our estimates of association, and by extension

437 parallelism, may be conservative if false-negatives are pervasive in high recombination

438 regions. Importantly however, our signatures of parallelism cannot be explained by variable

439 recombination.

$440 \quad$ Windows based on genetic distance $(0.1 \mathrm{cM})$ corroborated $50 \mathrm{~kb}$ results, returning

441 strong signals of parallelism for calcium, $\mathrm{pH}$, pelvic spine length, pelvis length, plate number

442 and gill raker number. Interestingly, we also recovered weakly significant parallelism for

443 several other environmental and armour variables (Supplementary Fig. 4, Supplementary

444 Information), suggesting potentially stronger parallelism than we report at 50kb windows.

445 To assess wider, linked parallel regions, we plotted 50kb windows across the genome

446 to examine clustering of all associated windows (Supplementary Fig. 5). Adjacent windows

447 (two or more, Supplementary Table 13) were pooled together to inspect putative causative

448 genes (Supplementary Dataset 2). Using these two methods we identified a number of

449 wider genomic regions that exhibited parallelism across multiple radiations. An example,

450 and good positive control for our method, involves the pooling of windows associated with

451 plate number in three radiations (Alaska, BC and Iceland) on chromosome IV around the

452 well-known Eda gene, which has a well-established role in producing variation in the

453 number of armour plates $\left({ }^{17,26,27}\right)$. This pattern emerges despite the limited variation in plate

454 number across freshwater populations.

455 Pooling adjacent windows also identified a large cluster (250 kb) on chromosome I

456 containing genes igfbp2a, stk11ip and atp1a1, and strongly associated with calcium, sodium 
457 and $\mathrm{pH}$ in several radiations (Supplementary Data 2). The clustering of windows in this

458 region is perhaps unsurprising given it contains a known inversion $\left({ }^{60}\right)$, which as discussed

459 should be beneficial for adaptive haplotypes by reducing local recombination $\left({ }^{61}\right)$. Within

460 this region specifically, it is likely that the atpa1a1 gene causes large effects on fitness, given

461 its previously detected association with the major ecological transition from marine to

462 freshwater $\left({ }^{62}\right)$ and functional role in metal ion management $\left({ }^{17,60}\right)$. Its apparent role in

463 adaptation to much smaller cation variation between freshwater environments in this study

464 is interesting in light of Fisher's geometric model of adaptation, since this predicts that only

465 alleles of smaller effect should fix as a fitness optimum is approached $\left({ }^{63}\right)$. Thus, it may be

466 that different mutations in this gene have a spectrum of effect sizes, or that changes cause

467 subtler differences in expression, rather than larger coding differences.

468 Our results support the existence of genomic regions of physically linked genes that 469 are hitch-hiking in separate radiation pairs, and may contain genes that are parallel across

470 all radiations but undetected by our genomic methods. Extensive linkage disequilibrium in

471 freshwater populations is consistent with what is expected under strong directional

472 selection after colonization from marine populations and has been reported for stickleback

473 populations from Alaska $\left({ }^{44}\right)$, but it had not previously been observed for the same regions

474 across several independent adaptive radiations. These results are also consistent with

475 previous findings of large numbers of SNPs highly divergent between marine and freshwater

476 stickleback aggregating in just 19 short genomic regions, including three known inversions

$477\left({ }^{60}\right)$, one of which we also detected and highlighted above. 
479 Relationships between genomic parallelism and phylogenetic, phenotypic and

480 environmental similarity.

481 Freshwater populations have radiated from marine common ancestors $\left({ }^{1,16}\right)$, thus the

482 parallelism patterns described in this study are putatively the result of multiple marine -

483 freshwater transitions. Based on this assumption, and using our SNP data from a marine

484 population within each radiation, we performed genome-wide $\mathrm{F}_{\text {ST }}$ outlier analyses

485 comparing each freshwater population with the marine population within that radiation.

486 We then took the top $5 \%$ of $50 \mathrm{~kb}$ windows according to $\mathrm{F}_{\mathrm{ST}}$ in each marine - freshwater

487 comparison and looked for overlapping outlier windows across all comparisons $(\mathrm{N}=2,628)$.

488 This quantified the extent of repeated genome-wide differentiation for MxF transitions

489 within and across all radiations (Fig. 4a).

We then used Mantel tests to test statistically the effects on MxF genomic

parallelism of relative genetic divergence and environmental/phenotypic similarity

492 alongside one another. We compared the matrix of overlapping marine - freshwater outlier

$493 \quad F_{S T}$ windows to equivalent matrices for environmental dissimilarity (Euclidean distances),

494 phenotypic dissimilarity (Euclidean distances), and genetic dissimilarity ( $F_{S T}$ values) (Fig. 4).

495 Across all population comparisons the number of parallel windows was strongly negatively

496 correlated with genetic dissimilarity $(r=-0.61, p<0.001)$, and also negatively correlated

497 with environmental dissimilarity $(r=-0.42, p<0.001)$ and phenotypic dissimilarity $(r=-0.11$,

$498 p=0.022$ ). These results highlight that genomic parallelism increases in populations that are

499 more genetically, environmentally and phenotypically similar, though to varying extents. 
$500 \quad$ Parallel windows were more common in intra- rather than inter-continental

501 comparisons. This highlights the significance of our European and North American pairings

502 as the major contributors towards pairwise signals of genomic parallelism, as we discussed

503 previously, and strongly suggests that genomic parallelism at large geographic scales is

504 contingent on shared genetic variation. Further, at an intra-continental scale, there exists

505 the possibility of haplotype sharing between radiations by gene flow through marine

506 populations, which may be facilitated in North America, despite the greater geographic

507 distance, by a shared coastline connecting Alaska and $\mathrm{BC}\left({ }^{64}\right)$. Recent research has also

508 highlighted a probable genetic bottleneck in the founding of Atlantic marine populations

509 that restricts shared freshwater alleles between Atlantic and Pacific freshwater populations

$510\left({ }^{39}\right)$

We also conducted partial mantel tests for the effects of environmental and

phenotypic similarity whilst controlling for genetic similarity, given this is likely to correlate

with environment and phenotype in some cases due to geographic proximity. Effects of

514 environmental dissimilarity were marginally reduced when controlling for genetic similarity,

515 but were still strongly negative $(r=-0.35, p<0.001)$, suggesting that similar environments

516 promote genomic parallelism irrespective of genetic similarity. Phenotypic dissimilarity was

517 no longer associated with genomic parallelism after controlling for genetic similarity $(r=-$

$5180.10, p=0.097)$. This latter result suggests that environmental similarity is a better predictor

519 of genomic parallelism than phenotypic similarity (at least in terms of observable

520 morphometric phenotypes) in this system.

521 In conclusion, our study is the largest to date in this system addressing the relative

522 effects of environment, phenotype and genetics in predicting parallel evolution. Genetic 
523 similarity is the best predictor of genomic parallelism here, in line with recent results and

524 expectations regarding sharing of ancestral variants and introgression between populations

$525\left({ }^{29,39}\right)$. However, even whilst controlling for this, environmental variation (most likely Ca and

$526 \mathrm{pH}$ variation in particular) is a good predictor of genome-wide parallelism among freshwater

527 populations on a wide geographic scale. In particular, the higher environmental similarity

528 among North American radiations, compared to European populations, provides a good

529 explanation for the strong phenotypic and genomic parallelism among those freshwater

530 populations, alongside the probable genetic bottleneck in the founding of Atlantic marine

531 populations $\left({ }^{39}\right)$. The fact that, despite the larger geographic distance between the two

532 North American radiations, they have more similar phenotypes and exhibit stronger

533 genomic parallelism demonstrates that environment, and by extension selection, can

534 counteract the effects of distance to some extent. Explicit measurements of environment

535 are thus important in predicting parallel evolution even across large geographic scales, and

536 distance alone can be misleading. Phenotypic parallelism was observed for several traits

537 across continents, and populations with similar phenotypes also exhibit stronger genomic

538 parallelism. However, this relationship is weaker than for commonality of environment, and

539 is likely confounded by variable heritability and genetic architecture among phenotypes.

540 Most importantly, our results highlight that quantitative analyses of phenotypes and

541 environments and of their relationship can provide a good prediction of expected genomic

542 parallelism, and provide a much clearer picture of major factors that are likely to influence

543 the emergence of a common pattern of genomic divergence than analyses of dichotomous

544 phenotypes and environments.

\section{Methods}




\section{Sampling and environmental data collection.}

\section{Population genetics statistics and phylogenetic tree.}

Raw sequence reads were demultiplexed using Stacks $-1.35\left({ }^{67}\right)$. Number of reads per individual are shown in Supplementary Dataset 3 (see Supplementary Information for details on the alignment of reads and Stacks pipeline used). For Bayenv2 data, autosomal SNPs were called as being in $>7$ populations, $>50 \%$ of the individuals within a population, and with a MAF-filter of 0.05. After filtering we retained 26,990, 26,937, 29,111, 26,169 SNPs for Scotland, Iceland, British Columbia and Alaska respectively. For analyses of population structure across all radiations, a subset of unlinked SNPS were generated. Here, autosomal SNPs were called that were present in all radiations and in $>50 \%$ of individuals within a radiation, with a MAF-filter of 0.05 (within a radiation). Only the first SNP per RAD locus was retained. $F_{S T}$ was bootstrapped and calculated in POPULATIONS. This set of SNPS were then pruned for linkage disequilibrium in plink using indep-pairwise 5050.2 . The set of unlinked SNPs were used to construct a neighbour-joining tree for all fish in the $R$ package 'adegenet', using a distance matrix computed from the SNP data $\left({ }^{68}\right)$. The tree was bootstrapped 100 times and nodes with less than $80 \%$ support were collapsed. The tree was plotted using the 'ape' package in R $\left({ }^{69}\right)$. PCA analysis of population structure was conducted using plink $\left({ }^{70}\right)$.

\section{Phenotypic and environmental variation - body shape, armour, gill rakers and environmental data analyses.}


591

All morphological measurements (body shape, body armour and spine traits) were done following $\left({ }^{25}\right)$. Details of the quantification of phenotypic traits can be found in Supplementary Information.

We performed three Principal Component Analyses (PCAs): one on the armour traits, another on body shape, and another on the 6 environmental variables. Body shape and armour PCAs were performed on regression residuals of all individuals from all radiations pooled together to extract the common PCs of body shape and armour variation (Shape_PCs and ArmourPCs) and environmental variation and retained axes that explained more than $10 \%$ of the total variance. Armour and environmental PCAs were conducted with scaled inputs due to different units of measurement between variables. Shape PCAs were conducted on morphometric residuals, and as such were not scaled. All phenotypic analyses, including ANOVAs and ANCOVAs and plotting were done in R version 3.4.3.

\section{Genotype-Environment/Phenotype Associations.}

For each radiation separately ( $N=18$ to 19 populations) we used Bayenv2 $\left({ }^{43}\right.$ ) to identify associations between genomic allele frequencies $(\mathrm{N}=10$ to 21 individual fish, mean= 17. 8), the set of six biotic and abiotic environmental variables ( $\mathrm{Ca}, \mathrm{Na}, \mathrm{pH}, \mathrm{Zn}$, prevalence of Gyrodactylus spp. and Schistochephalus solidus) and the set of 12 phenotypic traits (Shape_PC1, Shape_PC2, Shape_PC3, DS1, DS2, PS, LP, HP, BAP, Plate_N, Gill_Raker_L and Gill_Raker_N) mentioned above. For each radiation, a matrix of genetic covariance was calculated using a subset of SNPs limited to a single SNP per RAD-locus and pruned for linkage disequilibrium $\left(R^{2}<0.4\right)$ in plink $\left({ }^{70}\right)$. This cut-off was selected to balance the tradeoff between SNPs retained and minimising the effects of linkage. Covariance matrices were therefore calculated using 9619, 7983, 7300 and 5705 SNPs for Alaska, BC, Iceland and Scotland respectively. Covariance matrices were calculated across 100,000 iterations and averaged across 5 independent runs. Bayenv 2 was run independently 8 times and final results were averaged across runs.

Environmentally and phenotypically associated SNPS were selected as having a $\log _{10^{-}}$ BayesFactor > 1.5 and an absolute Spearman's rank coefficient above the 95th percentile. The combination of BayesFactor and non-parametric measure of correlation helps to avoid selecting SNPs with high BayesFactors due to spurious populations $\left({ }^{18}\right)$. SNPs were grouped into $50 \mathrm{~kb}, 75 \mathrm{~kb}, 100 \mathrm{~kb}, 200 \mathrm{~kb}$ and $0.1 \mathrm{cM}$ windows (Supplementary Table 14) to test the robustness of our results across different extents of linkage. To evaluate whether windows were environmentally or phenotypically associated, we adapted the methodology of $\left({ }^{46}\right)$. We calculated the upper $99 \%$ binomial expectation for the number of associated SNPs given the total number of SNPS in a specific window, and selected windows that had a greater number of associated SNPS than this expectation. This method controls for variation in SNP density across windows and ensures that significant windows exhibit consistent allele frequency correlations across multiple SNPs. We visualised the genomic locations of associated windows using Manhattan plots (Supplementary Fig. 5) and plotting the residual number of outlier SNPS above the binomial expectation (Supplementary Fig. 6). Linkage groups I-XXI were visualised with the exception of XIX; windows on scaffolds were not visualised. Finally, we compared these associated windows across radiations to examine those that were parallel.

\section{Parallelism statistics.}


637 For all radiation groupings (11 combinations in total: one four radiation grouping, four three

638 radiation groupings, and six two radiation groupings), we calculated the significance of

639 parallel window counts using a permutation method. For each environmental or phenotypic

640 variable, we randomly drew $\mathrm{N}$ windows from each radiation's total pool where $\mathrm{N}$ was

641 equivalent to the associated window count for each radiation. We then assessed the overlap

642 of randomly associated windows across radiations and pooled the results over 10,000

643 iterations. The output from all permutations was used as a null distribution to infer $\mathrm{p}$-values,

644 which were then FDR-corrected using the R package qualue $\left({ }^{71}\right)$.

645

Windows of $50 \mathrm{~kb}$ and above were based on a linkage assumption and to minimise nonindependence between windows. There were, however, occasionally adjacent windows associated with the same variable across different groupings. Large regions of relatively strong linkage are plausible if recombination is reduced through processes such as genomic rearrangements. To investigate these, we grouped associated windows that were adjacent as well as those that were direct matches across radiations based on the likelihood of adjacent associated windows resulting independently being low, suggesting nonindependence and probable linkage. These windows are available in Supplementary Table 13.

\section{Multivariate vector comparison of environments and phenotypes.} Vectors for environmental, shape and armour PCs ( $>10 \%$ variance) and gill raker data were calculated as the difference between the linearly predicted maximum and minimum values per radiation. Angles $(\theta)$ and difference in length $(\Delta L)$ were calculated for each vector between radiations. Significance of vectors was determined through permutations by simulating random traits from a normal distribution with mean and s.d. equivalent to the observed data and assessing vectors of random traits/variation as above (Supplementary Information).

\section{Comparing relative influences of environment, phenotype and genetics.}

$F_{S T}$ was calculated between each freshwater population and its relevant marine population (Alaska $=$ MUD1, BC = LICA, Iceland = NYPS, Scotland = OBSM) in 50kb windows using the R package 'PopGenome' (REF). For each MxF comparison, windows above the $95 \%$ quantile were classed as outliers. Outlier windows were compared across all pairwise freshwater comparisons (2,628 comparisons among 73 populations), with overlapping outliers representing $M x F F_{S T}$ parallelism. Dissimilarity matrices of environment and phenotype were calculated as Euclidean distance in PCA space for the 6 and 12 environmental and phenotypic variables respectively. The genetic dissimilarity matrix was composed of genome-wide pairwise $F_{S T}$ estimates between freshwater populations. The matrix of MxF parallelism was associated to environmental, phenotypic and genetic dissimilarity matrices using Mantel tests with 9,999 permutations. Partial Mantel tests were performed with genetic distance as the conditional matrix for environmental and phenotypic effects on MxF parallelism, again with 9,999 permutations.

\section{Acknowledgements}

We thank Shaun Robertson, Rebecca Young, Abdul Rahman, Brian Santos, Sara Goodacre, Petur Halldorsson, Bjarni K. Kristjánsson, Dolph Schluter, Kieran Samuk, Diana Rennison and Sara Miller for help with the sampling and sampling permits. We are grateful to Ann Lowe 684 and Laura Dean for help with the DNA extractions, to Cody Wiench, Amanda Stahlke and 
685

686

687

688

689

690

691

692

693

694

695

696

697

698

699

700

701

702

703

704

705

706

707

708

709

710

711

712

713

714

715

716

717

718

719

720

721

722

723

724

725

726

Sarah Hendricks for help making the RAD-libraries and to John Brookfield for discussion of probability calculations. This work was funded by a NERC grant (NE/J02239X/1 to A.D.C.M), and further support was provided by NIH grant P30GM103324.

\section{Contributions}

I.S.M, A.D.C.M and J.R.W. conceived the project, interpreted the data, and wrote the manuscript. I.S.M, D.D., and A.D.C.M performed field work. I.S.M, M.M. and D.D. generated the phenotypic data. I.S.M. and P.H. generated RAD data and J.R.W., I.S.M. and P.H. analysed it. P.H., M.B. and S.S. helped with the sampling and revised the manuscript.

\section{Competing financial interests}

The authors declare no competing financial interests.

\section{Data Accessibility}

Bam files of aligned reads for each individual and corresponding sample information have been deposited in the European Nucleotide Archive database under the project

PRJEB20851, with the sample accession numbers ERS1831811-ERS1833111, and run accession numbers ERR2055459-ERR2056759. Scripts used for all analyses are available at https://github.com/JimWhiting91/stickleback adaptive radiations/

\section{References}

1. Schluter, D. The ecology of adaptive radiation. (OUP Oxford, 2000).

2. Gavrilets, S. \& Losos, J. B. Adaptive radiation: Contrasting theory with data. Science 323, 732737 (2009).

3. Arnold, S. J., Bürger, R., Hohenlohe, P. A., Ajie, B. C. \& Jones, A. G. Understanding the

4. Losos, J. B. Adaptive radiation, ecological opportunity, and evolutionary determinism[:

5. Gould, S. J. Wonderful life: The Burgess shale and the nature of history. (New York: W W

6. Schluter, D. Adaptive Radiation Along Genetic Lines of Least Resistance. Evolution 50, 1766 (1996).

7. Roff, D. The evolution of the G matrix: Selection or drift? Heredity 84, 135-142 (2000).

8. Arendt, J., \& Reznick, D. Convergence and parallelism reconsidered: what have we learned

9. Stuart, Y. E. Divergent uses of "parallel evolution" during the history of the American naturalist. Am. Nat. 193, 11-19 (2019).

10. Mahler, D. L., Ingram, T., Revell, L. J. \& Losos, J. B. Exceptional convergence on the macroevolutionary landscape in island lizard radiations. Science 341, 292-295 (2013).

11. Oke, K. B., Rolshausen, G., LeBlond, C. \& Hendry, A. P. How parallel is parallel evolution? A comparative analysis in fishes. Am. Nat 190, 1-6 (2017).

12. Stuart, Y. E. et al. Contrasting effects of environment and genetics generate a continuum of 
parallel evolution. Nat. Ecol. Evol. 1, 0158 (2017).

13. Moser, D., Roesti, M. \& Berner, D. Repeated Lake-Stream Divergence in Stickleback Life History within a Central European Lake Basin. PLoS One 7, e50620 (2012).

14. MacColl, A. D. C. The ecological causes of evolution. Trends in Ecology and Evolution 26, 514522 (2011).

15. Elmer, K. R. \& Meyer, A. Adaptation in the age of ecological genomics: Insights from parallelism and convergence. Trends Ecol. Evol. 26, 298-306 (2011).

16. Conte, G. L., Arnegard, M. E., Peichel, C. L. \& Schluter, D. The probability of genetic parallelism and convergence in natural populations. Proc. R. Soc. B Biol. Sci. 279, 5039-5047 (2012).

17. Jones, F. C. et al. The genomic basis of adaptive evolution in threespine sticklebacks. Nature 484, 55-61 (2012).

18. Günther, T. \& Coop, G. Robust identification of local adaptation from allele frequencies. Genetics 195, 205-220 (2013).

19. Jacobs, A. et al. Convergence in form and function overcomes non-parallel evolutionary histories in Arctic charr. BioRxiv, 265272 (2018).

20. Paccard, A. et al. Repeatability of Adaptive Radiation Depends on Spatial Scale: Regional Versus Global Replicates of Stickleback in Lake Versus Stream Habitats. J. Hered. (2019).

21. Bell, M. A. \& Foster, S. A. The evolutionary biology of the threespine stickleback (Oxford University, 1994).

22. Taylor, E. B. \& McPhail, J. D. Historical contingency and ecological determinism interact to prime speciation in sticklebacks, Gasterosteus. Proc. R. Soc. B Biol. Sci. 267, 2375-2384 (2000).

23. Kaeuffer, R., Peichel, C. L., Bolnick, D. I. \& Hendry, A. P. Parallel and nonparallel aspects of ecological, phenotypic, and genetic divergence across replicate population pairs of lake and stream stickleback. Evolution 66, 402-418 (2012).

24. Ravinet, M., Prodöhl, P. A. \& Harrod, C. Parallel and nonparallel ecological, morphological and genetic divergence in lake-stream stickleback from a single catchment. J. Evol. Biol. 26, 186204 (2013).

25. Magalhaes, I. S., D'Agostino, D., Hohenlohe, P. A. \& MacColl, A. D. C. The ecology of an adaptive radiation of three-spined stickleback from North Uist, Scotland. Mol. Ecol. 25, 43194336 (2016).

26. Colosimo, P. F. et al. Widespread parallel evolution in sticklebacks by repeated fixation of ectodysplasin alleles. Science 307, 1928-1933 (2005).

27. Jones, F. C. et al. A genome-wide SNP genotyping array reveals patterns of global and repeated species-pair divergence in sticklebacks. Curr. Biol. 22, 83-90 (2012).

28. Raeymaekers, J. A. M. et al. Adaptive and non-adaptive divergence in a common landscape. Nat. Commun. 8, (2017).

29. Bassham, S., Catchen, J., Lescak, E., von Hippel, F. A. \& Cresko, W. A. Repeated selection of alternatively adapted haplotypes creates sweeping genomic remodeling in stickleback. Genetics 209, 921-939 (2018). 
30. Ferchaud, A. L. \& Hansen, M. M. The impact of selection, gene flow and demographic history on heterogeneous genomic divergence: Three-spine sticklebacks in divergent environments. Mol. Ecol. 25, 238-259 (2016).

31. Roesti, M., Moser, D. \& Berner, D. Recombination in the threespine stickleback genome Patterns and consequences. Mol. Ecol. 22, 3014-3027 (2013).

32. Schluter, D. \& Conte, G. L. Genetics and ecological speciation. Proc. Natl. Acad. Sci. 106, 9955-9962 (2009).

33. Renaut, S., Owens, G. L. \& Rieseberg, L. H. Shared selective pressure and local genomic landscape lead to repeatable patterns of genomic divergence in sunflowers. Mol. Ecol. 23, 311-324 (2014).

34. MacColl, A. D. C. \& Aucott, B. Inappropriate analysis does not reveal the ecological causes of evolution of stickleback armour: A critique of Spence et al. 2013. Ecol. Evol. 4, 3509-3513 (2014).

35. De Schamphelaere, K. A. C. et al. Reproductive toxicity of dietary zinc to Daphnia magna. Aquat. Toxicol. 70, 233-244 (2004).

36. Martins, C., Jesus, F. T. \& Nogueira, A. J. A. The effects of Copper and Zinc on survival, growth and reproduction of the cladoceran Daphnia longispina: introducing new data in an "old" issue. Ecotoxicology 26, 1157-1169 (2017).

37. Nelson, T. C. \& Cresko, W. A. Ancient genomic variation underlies repeated ecological adaptation in young stickleback populations. Evol. Lett. 2, 9-21 (2018).

38. Rennison, D. J., Delmore, K. E., Samuk, K., Owens, G. L. \& Miller, S. E. Shared patterns of genome-wide differentiation are more strongly predicted by geography than by ecology. (2019). doi:10.1086/706476

39. Fang, B., Kemppainen, P., Momigliano, P., \& Merilä, J. Oceans apart: Heterogeneous patterns of parallel evolution in sticklebacks. BioRxiv, 826412 (2019).

40. Mäkinen, H. S. \& Merilä, J. Mitochondrial DNA phylogeography of the three-spined stickleback (Gasterosteus aculeatus) in Europe-Evidence for multiple glacial refugia. Mol. Phylogenet. Evol. 46, 167-182 (2008).

41. Liu, S., Hansen, M. M. \& Jacobsen, M. W. Region-wide and ecotype-specific differences in demographic histories of threespine stickleback populations, estimated from whole genome sequences. Mol. Ecol. 25, 5187-5202 (2016).

42. Fang, B., Merilä, J., Ribeiro, F., Alexandre, C. M. \& Momigliano, P. Worldwide phylogeny of three-spined sticklebacks. Mol. Phylogenet. Evol. 127, 613-625 (2018).

43. Coop, G., Witonsky, D., Di Rienzo, A. \& Pritchard, J. K. Using environmental correlations to identify loci underlying local adaptation. Genetics 185, 1411-1423 (2010).

44. Hohenlohe, P. A. et al. Population genomics of parallel adaptation in threespine stickleback using sequenced RAD tags. PLoS Genet. 6, e1000862 (2010).

45. Guo, B., DeFaveri, J., Sotelo, G., Nair, A. \& Merilä, J. Population genomic evidence for adaptive differentiation in Baltic Sea three-spined sticklebacks. BMC Biol. 13, 19 (2015).

46. Yeaman, S. et al. Convergent local adaptation to climate in distantly related conifers. Science 353, 1431-1433 (2016). 
47. Miller, C. T. et al. Modular skeletal evolution in sticklebacks is controlled by additive and clustered quantitative trait loci. Genetics 197, 405-420 (2014).

48. Chan, Y. F. et al. Adaptive evolution of pelvic reduction in sticklebacks by recurrent deletion of a pitxl enhancer. Science 327, 302-305 (2010).

49. Glazer, A. M., Cleves, P. A., Erickson, P. A., Lam, A. Y. \& Miller, C. T. Parallel developmental genetic features underlie stickleback gill raker evolution. Evodevo 5, 19 (2014).

50. Day, T., Pritchard, J., \& Schluter, D. A comparison of two sticklebacks. Evolution, 48(5), 17231734 (1994).

51. Garduno-Paz, M. V, Couderc, S. \& Adams, C. E. Habitat complexity modulates phenotype expression through developmental plasticity in the threespine stickleback. Biol. J. Linn. Soc. 100, 407-413 (2010).

52. McCairns, R. J. S. \& Bernatchez, L. Plasticity and heritability of morphological variation within and between parapatric stickleback demes. J. Evol. Biol. 25, 1097-1112 (2012).

53. Marques, D. A. et al. Genomics of Rapid Incipient Speciation in Sympatric Threespine Stickleback. PLoS Genet. 12, (2016).

54. Lowry, D. B. et al. Breaking RAD: an evaluation of the utility of restriction site-associated DNA sequencing for genome scans of adaptation. Mol. Ecol. Resour. 17, 142-152 (2017).

55. McKinney, G. J., Larson, W. A., Seeb, L. W. \& Seeb, J. E. RADseq provides unprecedented insights into molecular ecology and evolutionary genetics: comment on Breaking RAD by Lowry et al. (2016). Mol. Ecol. Resources 17, 356-361 (2017).

56. Roesti, M., Kueng, B., Moser, D. \& Berner, D. The genomics of ecological vicariance in threespine stickleback fish. Nat. Commun. 6, (2015).

57. Catchen, J. M. et al. Unbroken: RADseq remains a powerful tool for understanding the genetics of adaptation in natural populations. Mol. Ecol. Resources 17, 362-365 (2017).

58. Samuk, K. et al. Gene flow and selection interact to promote adaptive divergence in regions of low recombination. Mol. Ecol. 26, 4378-4390 (2017).

59. Meier, J. I., Marques, D. A., Wagner, C. E., Excoffier, L. \& Seehausen, O. Genomics of parallel ecological speciation in Lake Victoria cichlids. Mol. Biol. Evol. 35, 1489-1506 (2018).

60. Terekhanova, N. V. et al. Fast Evolution from Precast Bricks: Genomics of Young Freshwater Populations of Threespine Stickleback Gasterosteus aculeatus. PLoS Genet. 10, e1004696 (2014).

61. Ravinet, M. et al. Interpreting the genomic landscape of speciation: a road map for finding barriers to gene flow. Journal of Evolutionary Biology 30, 1450-1477 (2017).

62. Shimada, Y., Shikano, T. \& Merilä, J. A high incidence of selection on physiologically important genes in the three-spined stickleback, gasterosteus aculeatus. Mol. Biol. Evol. 28, 181-193 (2011).

63. Fisher, R. A. The genetical theory of natural selection, 1st edn. Clarendon. (1930).

64. Ken A. Thompson, Osmond, M. M. \& Schluter, D. Patterns of speciation and parallel genetic evolution under adaptation from standing variation. biorxiv.org 18, 292-296 (2010).

65. Etter, P. D., Preston, J. L., Bassham, S., Cresko, W. A. \& Johnson, E. A. Local de novo assembly of rad paired-end contigs using short sequencing reads. PLoS One 6, e18561 (2011). 
850 66. Ali, O. A. et al. Rad capture (Rapture): Flexible and efficient sequence-based genotyping. Genetics 202, 389-400 (2016).

67. Catchen, J., Hohenlohe, P. A., Bassham, S., Amores, A. \& Cresko, W. A. Stacks: An analysis tool set for population genomics. Mol. Ecol. 22, 3124-3140 (2013).

68. Jombart, T. Adegenet: A R package for the multivariate analysis of genetic markers. Bioinformatics 24, 1403-1405 (2008).

69. Paradis, E., Claude, J. \& Strimmer, K. APE: Analyses of phylogenetics and evolution in R language. Bioinformatics 20, 289-290 (2004).

70. Chang, C. C. et al. Second-generation PLINK: Rising to the challenge of larger and richer datasets. Gigascience 4 (2015).

71. Storey, J. qualue: Q-Value estimation for false discovery rate control. R package version 2.0.0. 
bioRxiv preprint doi: https://doi.org/10.1101/856344; this version posted November 29,2019 . The copyright holder for this preprint (which was not certified by peer review) is the author/funder, who has granted bioRxiv a license to display the preprint in perpetuity. It is made available under aCC-BY-NC-ND 4.0 International license.

864

865

866

867

868

869
Fig. 1. Sampling sites and bootstrapped NJ tree for stickleback from 73 freshwater populations from four countries on two continents, based on 8,395 genetic markers for 1,304 individuals. All nodes shown have bootstrap support of at least 80 (other nodes were collapsed). Branches are coloured by the radiation to which they belong. Tips represent individual fish, which were generally tightly clustered by population (small labels). Stars represent lakes sampled.

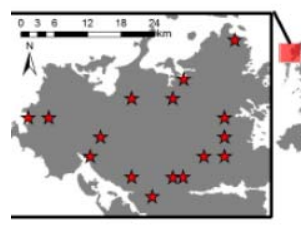

SCOTLAND

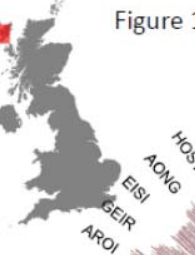

Figure 1.

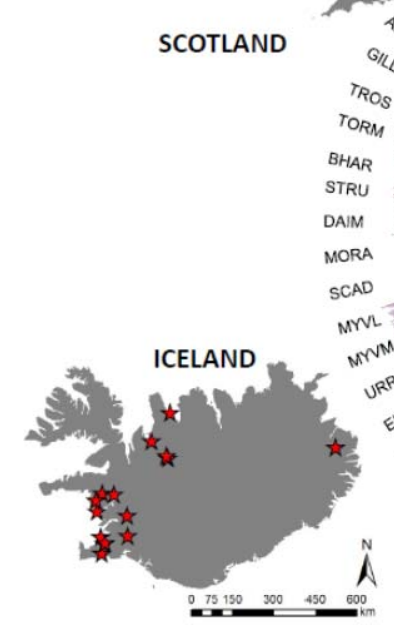

$1 / 4$
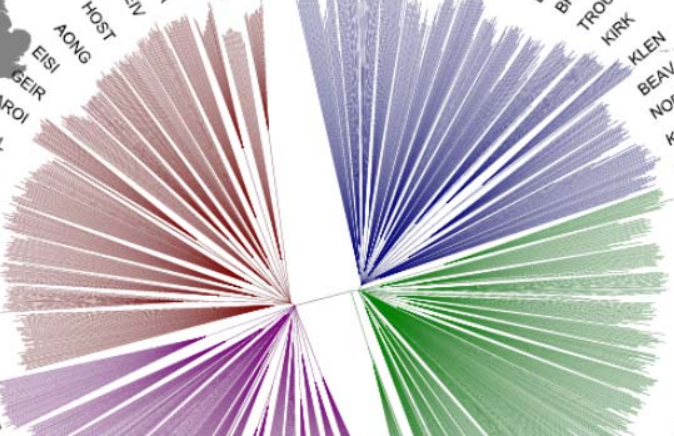

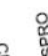 \\ \%}
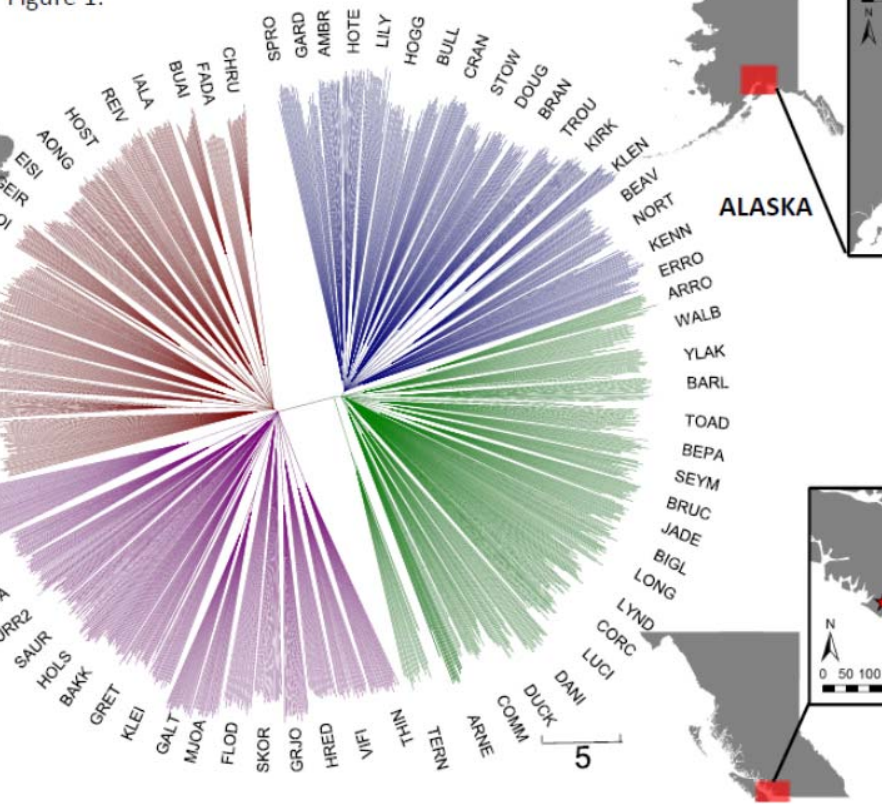

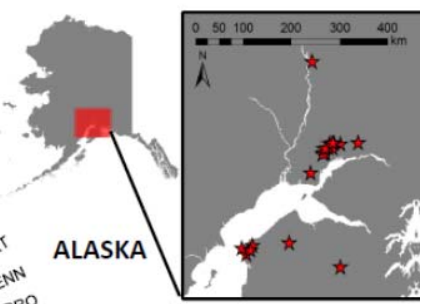

ERRO

WALB

YLAK

BARL.

TOAD

$B E P A$

BEPA

SEYM

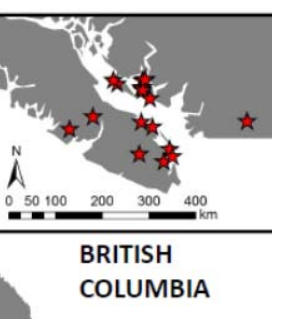

871 
873

Fig. 2. Principal Component Analyses of environmental variables (Environment); regression residuals of Procrustes coordinates against log centroid of body shape (Shape); armour traits (Armour: length of dorsal spines 1 (DS1) and 2 (DS2), length of pelvic spine (PS), length and height of pelvis (LP and $\mathrm{HP}$ ), length of biggest armour plate (BAP) and number of armour plates (Plate_N); and gill raker numbers (Gill_Raker_N) vs gill raker length (Gill_Raker_L) (Trophic). Each point represents a population and ellipses are $95 \%$ confidence ellipses. Names of variables with the highest positive (+) or negative (-) loadings on each axes are on legends of each axes. All loadings of variables on the first 3 PCs are in Supplementary Table 3. Marine populations ( + ) are projected where data was available using PC loadings calculated with freshwater populations only.

\section{Radiation - Alaska $\Delta$ BC Iceland + Marine $\otimes$ Scotland}

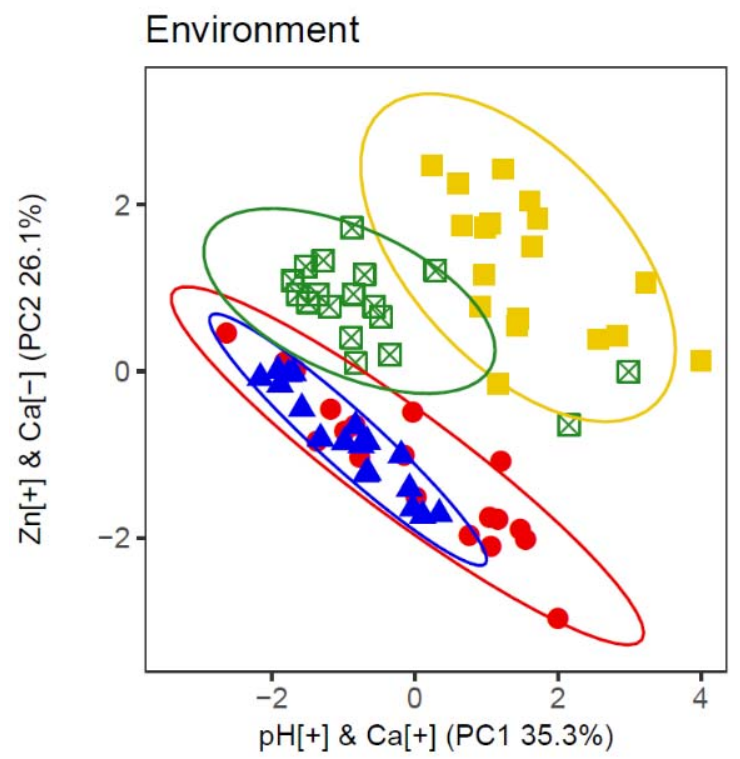

Shape

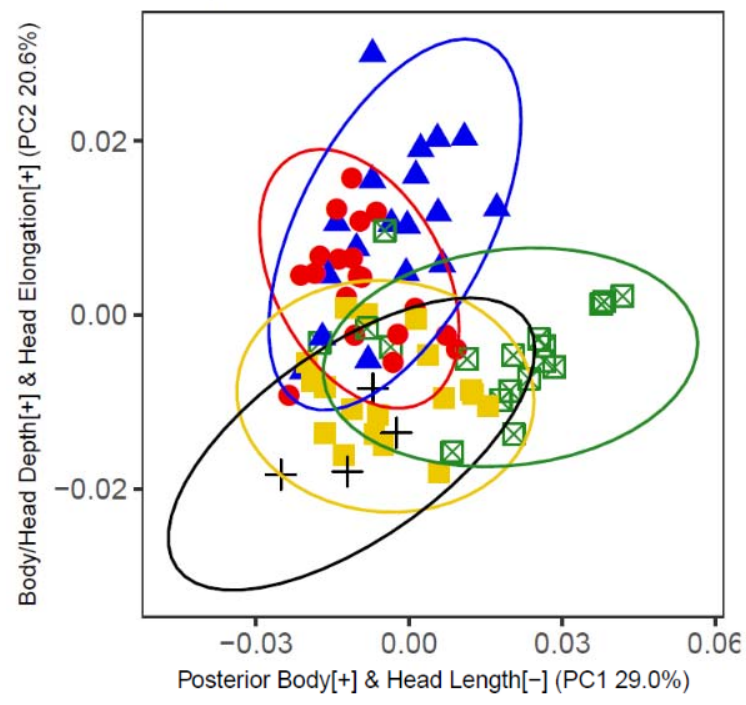

\section{Armour}

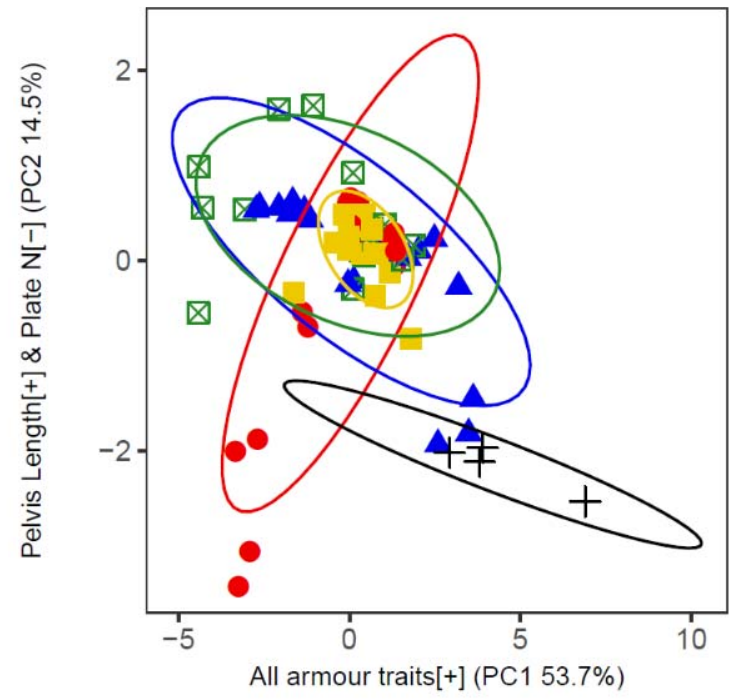

Trophic

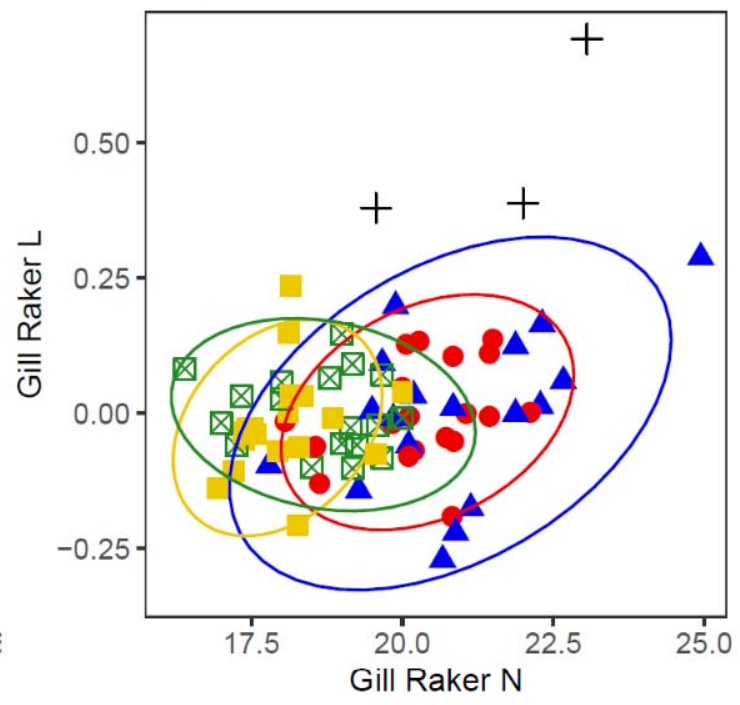


bioRxiv preprint doi: https://doi.org/10.1101/856344; this version posted November 29,2019 . The copyright holder for this preprint (which was not certified by peer review) is the author/funder, who has granted bioRxiv a license to display the preprint in perpetuity. It is made available under aCC-BY-NC-ND 4.0 International license.

885

886

887

888

889

890

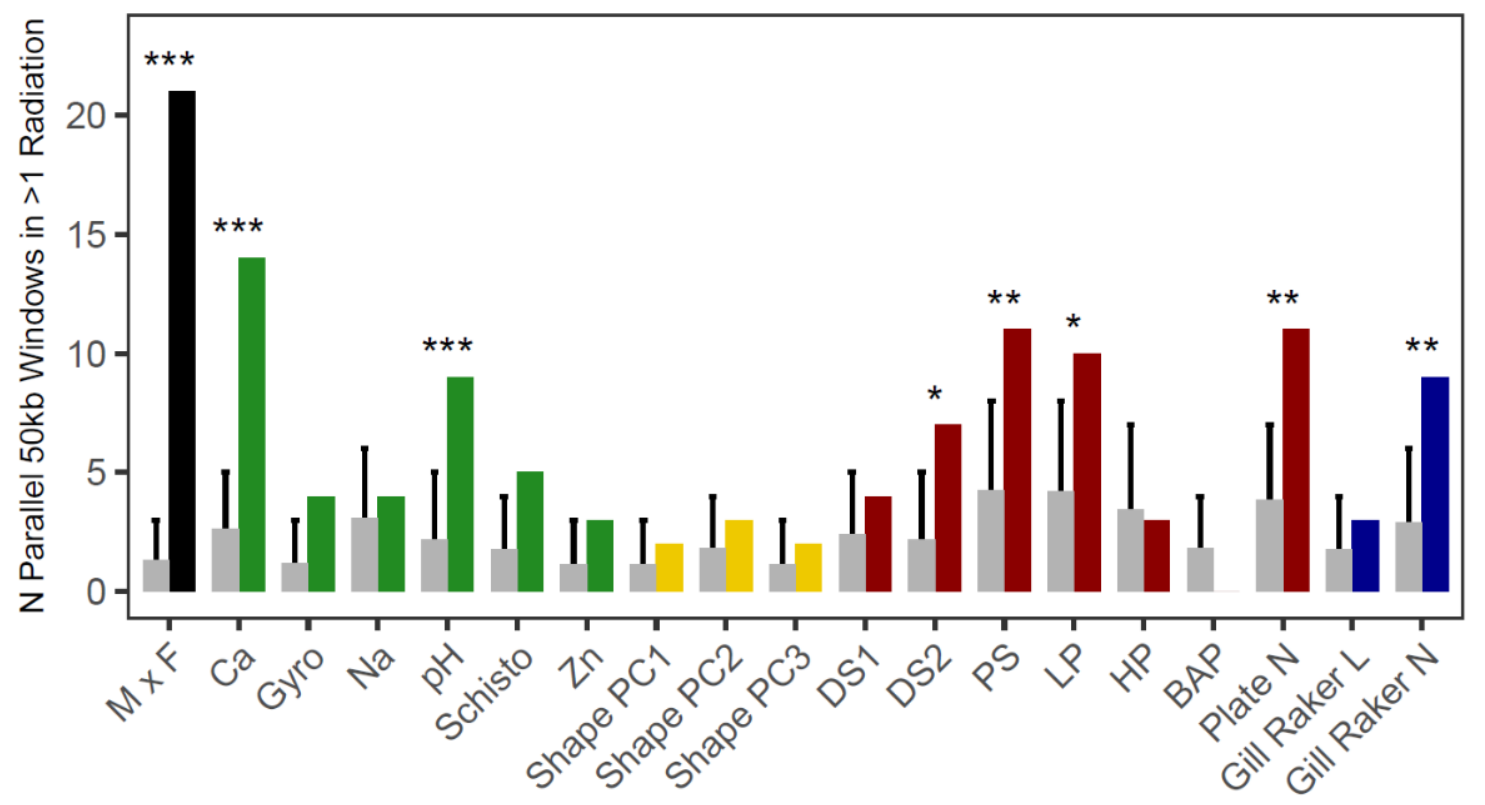

Fig. 3. Expected and observed counts of $50 \mathrm{~kb}$ windows containing an above $99 \%$ binomial expectation number of SNPs associated with marine $x$ freshwater (MxF), environmental variables and phenotypic traits in at least 2 radiations. Expected bars (grey) represent mean counts across 10,000 simulated outcomes with $95 \%$ confidence intervals per a one-tailed hypothesis. Asterisks denote significance of FDR-corrected one-tailed tests between the observed counts and the 100,000 simulated counts at the $<0.05\left({ }^{*}\right),<0.01(* *)$ and $<0.001(* * *)$ levels. 
bioRxiv preprint doi: https://doi.org/10.1101/856344; this version posted November 29, 2019. The copyright holder for this preprint (which was not certified by peer review) is the author/funder, who has granted bioRxiv a license to display the preprint in perpetuity. It is made available under aCC-BY-NC-ND 4.0 International license.

893 Fig. 4. Associations between genome-wide marine - freshwater $F_{S T}$ and environmental, phenotypic 894 and genetic distance across all pairwise comparisons of 73 freshwater populations. A) Proportion of $895 \mathrm{MxF}_{\text {ST }}$ 50kb outlier windows that overlap among freshwater replicates. Freshwater populations are 896 ordered as Alaska, British Columbia, Iceland and Scotland, with these location distinguishable as four 897 clear clusters. B) Environmental distance between freshwater populations, recorded as euclidean 898 distance in PCA space for all 6 environmental variables. C) Phenotypic distance between freshwater populations, recorded as for environmental distance for the 12 phenotypic variables. D) Genetic distance between freshwater populations, recorded as genome-wide pairwise $F_{S T}$ based on 8,395 unlinked SNPs. E-G) Associations between environmental (E), phenotypic (F) and genetic ( $G$ ) distance and $\mathrm{MxF}_{\mathrm{ST}}$ overlap (log-transformed). Points are coloured according to whether pairwise comparison is being made within a radiation or across radiations.

A

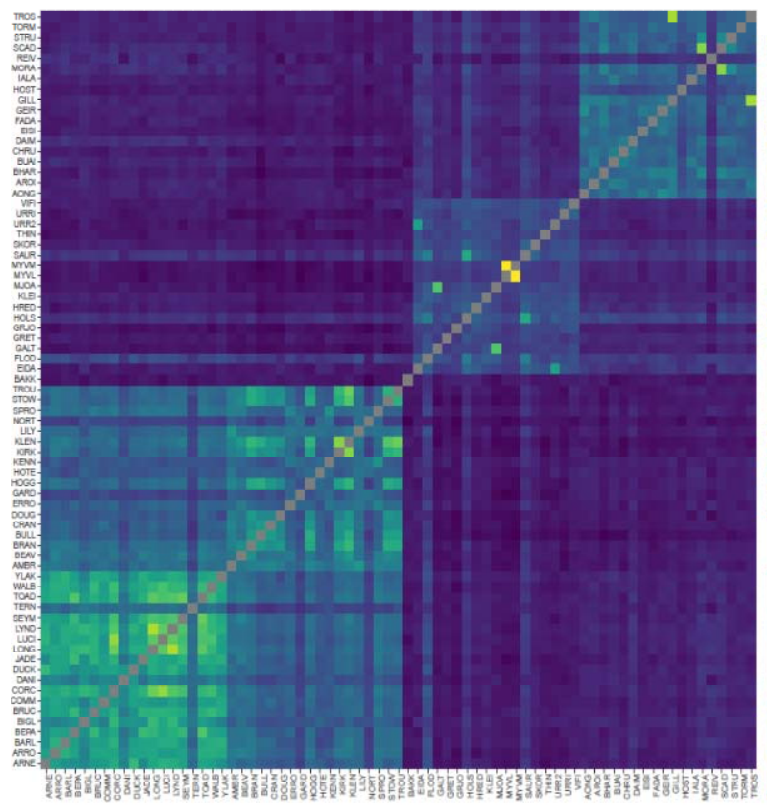

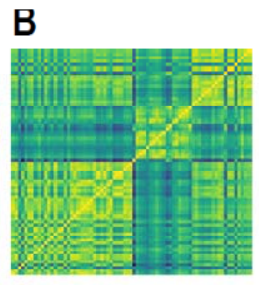

C

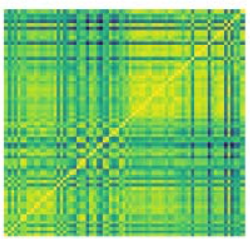

D

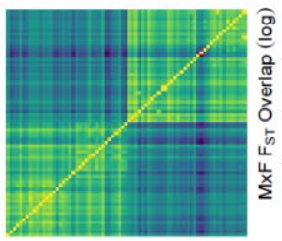

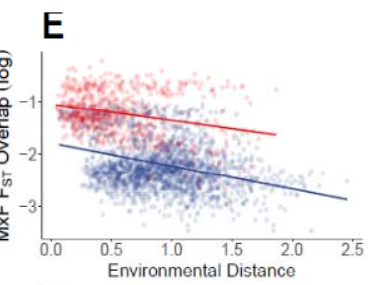

$F$

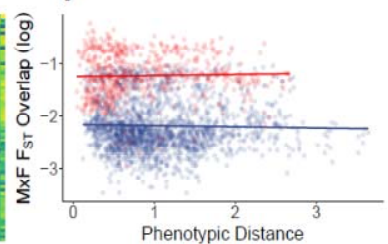

G

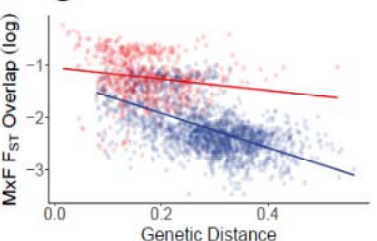

